\title{
Micro-welding of High Thermal Conductive Material Aluminum-Graphite Composite by Pulsed Nd:YAG Laser
}

\author{
Mohd Idris Shah ISMAIL ${ }^{* 1}$, Yasuhiro OKAMOTO*1 ${ }^{*}$ Akira OKADA*1 ${ }^{*}$ Yoshiyuki UNO ${ }^{* 1}$ and Muhaizad MUKHTAR ${ }^{* 1}$ \\ ${ }^{* 1}$ Graduate School of Natural Science and Technology, Okayama University, \\ 3-1-1 Tsushimanaka, Kita-ku, Okayama 700-8530, Japan \\ E-mail: idris@ntmlab.mech.okayama-u.ac.jp
}

\begin{abstract}
The development of advanced materials with superior high thermal properties and high specific strength has led to new metal matrix composites (MMCs) as a great attractive material in electrical and electronic industries. In order to manufacture more practical component from MMCs, a technique for joining MMCs to other similar composites or monolithic materials is strongly required. Therefore, the reliable and economic joining technique is investigated to increase the applications of MMCs. In this study, the overlap welding of pure aluminum and super thermal conductive (STC) aluminum-graphite composite was experimentally and numerically investigated by using a pulsed $\mathrm{Nd}$ :YAG laser. In order to discuss the welding of dissimilar materials with different thermophysical properties, the temporal change of heat input was controlled by arranging the laser pulse waveform. The porosities and bumps were observed as the remarkable weld defects in the welding process without a pulse control. On the other hand, the weld bead was largely free of defects, and a size of bump was relatively small with the appropriate controlled pulse waveform. It was clarified that the laser welded joint of an aluminum and a STC aluminum-graphite composite could be successfully achieved with the better weld penetration stability by the appropriate controlled pulse waveform.

DOI:10.2961/jlmn.2013.01.0018
\end{abstract}

Keywords: super thermal conductive, aluminum-graphite, overlap welding, pulse waveform

\section{Introduction}

The heat would be diffused from electronic devices by passive strategies, which carried out by the use of high thermal conductivity materials as a heat sink. The development of advanced materials with the superior high thermal properties and high specific strength has led to new metal matrix composites (MMCs) as a great attractive material in the electrical and electronic industries. Aluminum and its alloys are widely used for the manufacturing of MMCs, which have reached the industrial stage at some areas [1]. In order to manufacture practical components from MMCs, a technique for joining MMCs to other similar composites or to monolithic materials is strongly required. Therefore, the development of reliable and economic joining technique is investigated for extending the applications of MMCs. It is well-known that laser welding is the most flexible and versatile welding technology, and it has succeeded in the welding of MMCs [2, 3].

Recently, a super thermal conductive (STC) aluminumgraphite (Al-Gr) composite with the high thermal conductivity and the low thermal expansion coefficient was developed [4]. The properties of thermal conductivity versus thermal expansion coefficient are summarized in Figure 1, where the upper ellipse zone shows the STC composite materials, and the other of conventional thermal conductive materials such as $\mathrm{Cu}, \mathrm{Al}, \mathrm{Al}-\mathrm{SiC}, \mathrm{Cu}-\mathrm{W}, \mathrm{Cu}-\mathrm{Mo}, \mathrm{AlN}$ and $\mathrm{Si}$ are also indicated. It is difficult to weld STC Al-Gr composite material compared with existing MMCs, since the graphite material only can be melted under the high pressure with the high temperature [5]. In the laser welding, the use of standard pulse profile is limited to joint this ma- terial, since the uncontrolled heat input generates an overshoot, which leads to undesirable welded joints. The control of heat input by a pulse waveform was very important to achieve a suitable welding penetration, preventions of overheating condition and unacceptable welding defects.

In this study, the overlap welding of aluminum and STC aluminum-graphite composite was experimentally and numerically investigated by using a pulsed Nd:YAG laser beam with the control of pulse waveforms, which can provide a well-controlled heat input with high energy density. These investigations have led to an optimum welding condition proposed for a pulsed laser welding with minimum weld defects. The overlap welding of pure aluminum and STC Al-Gr composite was carried out, and the welding characteristics of STC Al-Gr composite were experimental-

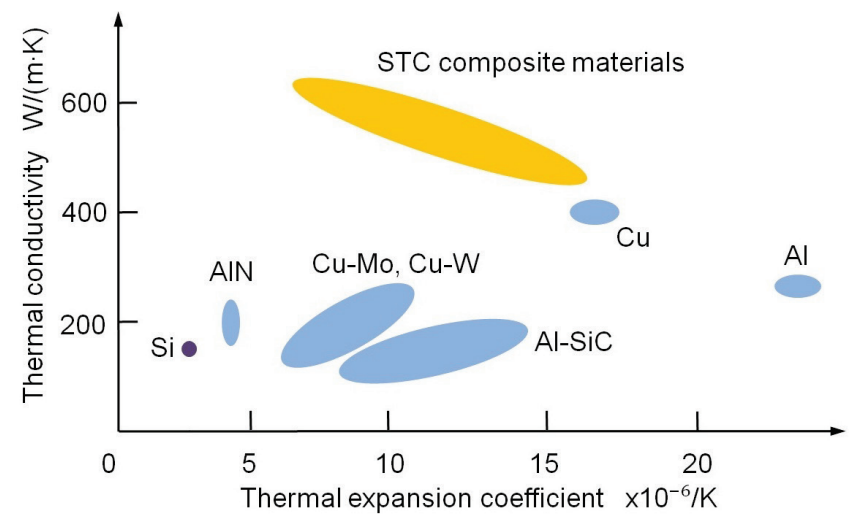

Fig. 1 Thermal conductivity and thermal expansion coefficient of STC composites and other materials. 
Table 1 Material properties of STC Al-Gr composite [6]

\begin{tabular}{ll}
\hline Material properties & Value \\
\hline Thermal conductivity $k(x x), k(y y)$ & $450 \mathrm{~W} /(\mathrm{m} \cdot \mathrm{K})$ \\
$k(z z)$ & $40 \mathrm{~W} /(\mathrm{m} \cdot \mathrm{K})$ \\
Specific heat $c$ & $810 \mathrm{~J} /(\mathrm{kg} \cdot \mathrm{K})$ \\
Density $\rho$ & $2450 \mathrm{~kg} / \mathrm{m}^{3}$ \\
Thermal expansion coefficient \\
$\quad \alpha(x x), \alpha(y y)$ & $17 \times 10^{-6} / \mathrm{K}$ \\
$\alpha(z z)$ & $8 \times 10^{-6} / \mathrm{K}$ \\
Bending strength $M(x x)$ & $65 \mathrm{MPa}$ \\
$M(z z)$ & $7 \mathrm{MPa}$ \\
Tensile strength $\sigma$ & $49 \mathrm{MPa}$ \\
Young modulus $G$ & $31 \mathrm{GPa}$ \\
\hline
\end{tabular}

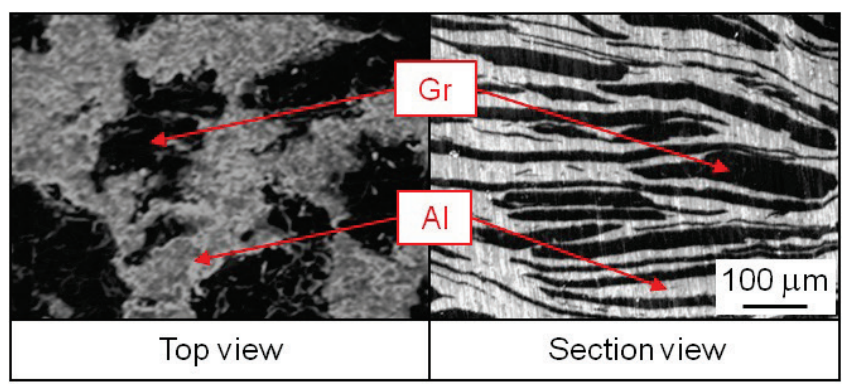

Fig. 2 Optical micrographs of STC Al-Gr composite.

ly investigated by the observation of joint part with optical microscope and scanning electron microscope (SEM). Moreover, the temperature distributions in the laser microwelding process were numerically analyzed to discuss the proper heat input. The weld strength was also evaluated by a shear test for the overlap welding with and without the control of pulse waveform.

\section{Experimental procedures}

The STC Al-Gr composite was used as the main specimen, and its material properties are shown in Table 1. Figure 2 shows the orientation of the Al-Gr composite in $x y$ direction (top view) and $x z$-direction (section view) before any laser irradiation. The specimens have been prepared to realize the properties of higher thermal conductivity and lower thermal expansion coefficient by utilizing a pulsed electric current sintering method [4].

A schematic diagram of experimental setup is shown in Figure 3. In this study, a pulsed Nd:YAG laser (LASAG SLS200 CL8) of $1064 \mathrm{~nm}$ in wavelength was used as a laser source. The laser beam was collimated to $15 \mathrm{~mm}$ in diameter and delivered by an optical fiber of $50 \mu \mathrm{m}$ core diameter. The collimator was installed between the optical fiber and the bending mirror, and the focusing point was coordinated by a lens of $50 \mathrm{~mm}$ in focal length. In order to avoid the back-reflection of incident laser beam, the processing head was aligned by 10 degrees to the perpendicular axis of the specimen surface. The experiments were carried out in the overlap welding, where the $0.3 \mathrm{~mm}$ thickness of pure aluminum sheet mounted on an Al-Gr

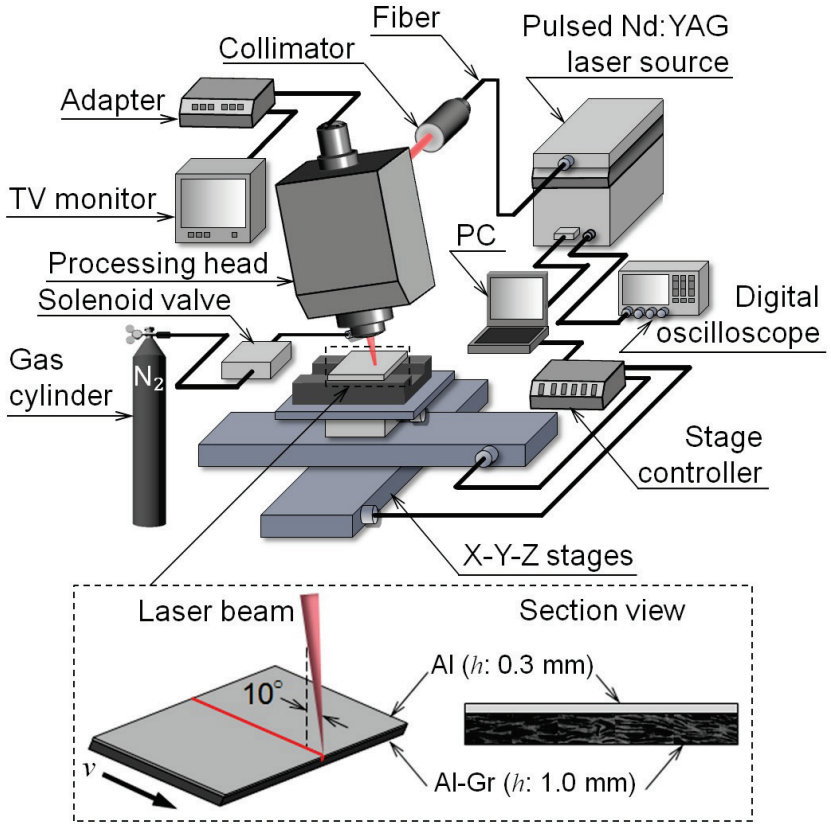

Fig. 3 Schematic diagram of experimental setup.

composite with $1.0 \mathrm{~mm}$ thickness. The welding experiments were done in a shielding gas of nitrogen with $13 \mathrm{~L} / \mathrm{min}$ flow rate. The stage controller could determine the movement of $\mathrm{X}-\mathrm{Y}-\mathrm{Z}$ stage, and it also synchronized the movement with the laser pulse.

After the laser welding, the sectioned surface of welded specimen was ground, polished and etched for the observation of weld bead by an optical microscope and SEM. In addition, the shear test was carried out to measure the shear strength of the overlap welded joints with a Shimadzu EZ$\mathrm{L}$ test machine. The cross-head speed was set to 0.5 $\mathrm{mm} / \mathrm{min}$. The specimen was gripped by the clampers, which are placed in the fixture blocks. Then, a shear load was slowly increased at the suitable increments by the mechanical lever system until the welded joint of specimen was fractured.

\section{Numerical analysis}

In this study, the further analysis of the welding phenomenon was conducted by the heat conduction analysis with finite element method (FEM). The analysis model is based on the fundamental heat transfer for the laser welding process. Based on the first law of thermodynamics, the equation for heat flow in a three-dimensional solid can be written as equation (1) [7]

$$
\rho \cdot c(\theta) \frac{\delta \theta}{\delta t}=\frac{\delta}{\delta x}\left[k(\theta) \frac{\delta \theta}{\delta x}\right]+\frac{\delta}{\delta y}\left[k(\theta) \frac{\delta \theta}{\delta y}\right]+\frac{\delta}{\delta z}\left[k(\theta) \frac{\delta \theta}{\delta z}\right]+Q_{v}
$$

where $\rho, c(\theta), k(\theta)$ and $Q_{v}$ are the material density, the temperature dependent specific heat, the temperature dependent thermal conductivity and the volumetric heat source term which varies with a laser power. $\theta=\theta(x, y, z, t)$ is the resulting three-dimensional and temporal temperature distribution in the material. $t$ is time, and $x, y, z$ are the spatial Cartesian coordinates.

Figure 4 shows the developed finite element model, and also shows the magnified mesh view near the interface. Fine mesh resolution is given at and near the heat source, while a fairly coarse mesh density is considered at the re- 
JLMN-Journal of Laser Micro/Nanoengineering Vol. 8, No. 1, 2013

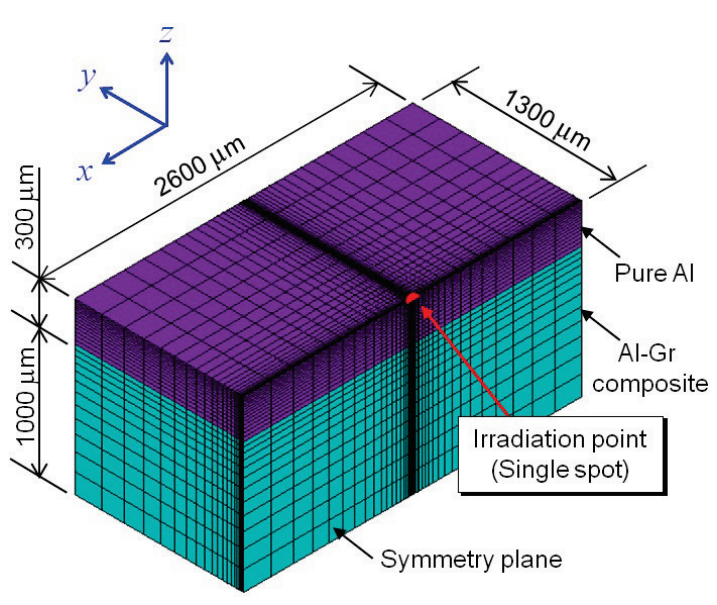

Fig. 4 Finite element model for heat conduction analysis.

gion far from the heat source. A portion of the specimens was designed in the analysis model in order to reduce the calculation time. In addition, the half model with symmetric conditions is used in the analysis. As the heat source of laser beam is symmetric in the $x-z$ plane, only half the heat source is considered. The heat source comprises a Gaussian plane heat source on the top surface and a conical shape heat source along the thickness of the specimen. To simplify the analysis, it was assumed that the alignment of laser spot was perpendicular to the specimen surface and a single spot of laser irradiation was utilized in the heat conduction analysis. The convective heat transfer condition of air was considered after the set time of laser irradiation. Except for a laser beam irradiated area, the convective heat transfer condition of air was also assumed. The main FEM analytical conditions are shown in Table 2.

\section{Results and discussions}

\subsection{Rectangular pulse waveform}

The variations of laser power from 300 to $550 \mathrm{~W}$ with constant $1 \mathrm{~ms}$ pulse width were irradiated on the specimen of $0.3 \mathrm{~mm}$ thickness aluminum and $1.0 \mathrm{~mm} \mathrm{Al-Gr}$ composite plate as shown in Figure 5. It could be seen that the laser pulse energy less than $400 \mathrm{~mJ} /$ pulse was insufficient to form a molten geometry for joining both materials, in which the penetration depth was shallow. The higher pulse energy was crucial to achieve the sufficient penetration depth and control the formation of molten geometry. However, the cross-section view above $450 \mathrm{~mJ} /$ pulse showed signs of porosity and bump defects. Moreover, the underfill defect was observed at the higher pulse energy of 550 $\mathrm{mJ} /$ pulse. Judging from these observation results, it was confirmed that the higher laser power (more than $400 \mathrm{~W}$ ) causes destructive effects, and lower laser power (less than $400 \mathrm{~W})$ will restrict the penetration depth and joining. From these viewpoints mentioned above, the next welding experiments were conducted under the constant $400 \mathrm{~W}$ laser power with various pulse widths in the rectangular pulse waveform.

Figure 5 shows welding results with the rectangular pulse waveform for various pulse widths and laser powers. As shown in the figure, the width of laser pulses had a significant role on the penetration depth. The weld joint between aluminum plate and Al-Gr composite can be seen at
Table $2 \quad$ FEM analysis conditions

\begin{tabular}{ll}
\hline Parameter/condition & Value \\
\hline Laser power $P_{\mathrm{p}}$ & $400 \mathrm{~W}$ \\
Pulse width $\tau$ & $2.7,3.0,5.0 \mathrm{~ms}$ \\
Beam diameter $d$ & $50 \mu \mathrm{m}$ \\
Heat transfer coefficient $h$ & $10 \mathrm{~W} /\left(\mathrm{m}^{2} \cdot \mathrm{K}\right)$ \\
Room temperature $\theta$ room & $293 \mathrm{~K}$ \\
\hline
\end{tabular}

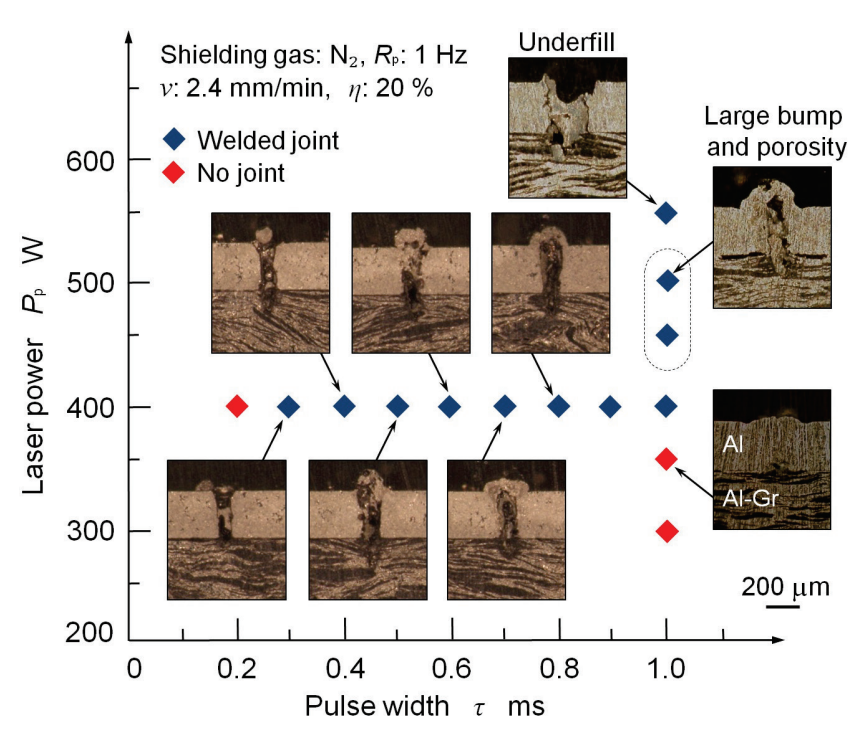

Fig. 5 Welding results with rectangular pulse waveform.

the pulse widths more than $0.3 \mathrm{~ms}$. The penetration depth and bead width gradually increased with increasing the pulse width. However, these increments led to the increase of bump size in the weld joint. On the other hand, the penetration depth increased gradually with increasing the laser pulse energy, and porosity and bump defects were observed in a joining part between pure aluminum plate and Al-Gr composite. According to the observation results of crosssection in the welding with rectangular pulse waveform, the pulse width of $0.6 \mathrm{~ms}$ was better condition with the deeper penetration, smaller porosities and bumps.

\subsection{Controlling pulse waveform}

As mentioned in the observation results of uncontrolled pulse waveform mentioned above, the bump and porosity defects were the major problem in the overlap welding between pure aluminum and Al-Gr composite. In order to overcome these problems, a controlled pulse waveform is discussed, since it is considered that an appropriate controlled laser pulse waveform could generate a better welded joint. Figure 6 shows the controlled pulse waveform named as a spike pulse waveform. The spike pulse waveform is divided into two phases. At the phase 1, the laser power of $400 \mathrm{~W}$ and pulse width of $0.6 \mathrm{~ms}$ were selected according to the previous experimental results of rectangular pulse waveform. Phase 2 is a subsequent function of the phase one by adding the heat to melt the bump generated in the phase one. This re-melting process is intended to remove the porosity in order to obtain a better joining state. Therefore, the experiments were carried out to discuss an appro- 


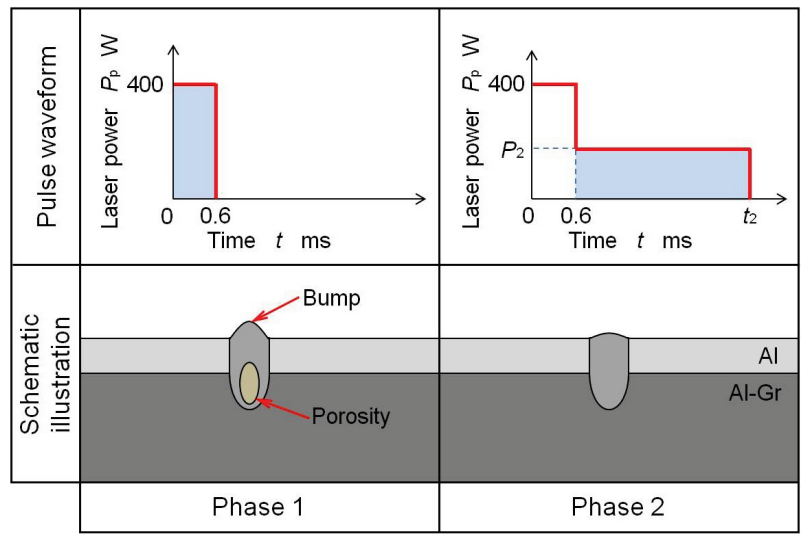

Fig. 6 Schematic illustration of welding process with spike pulse waveform.

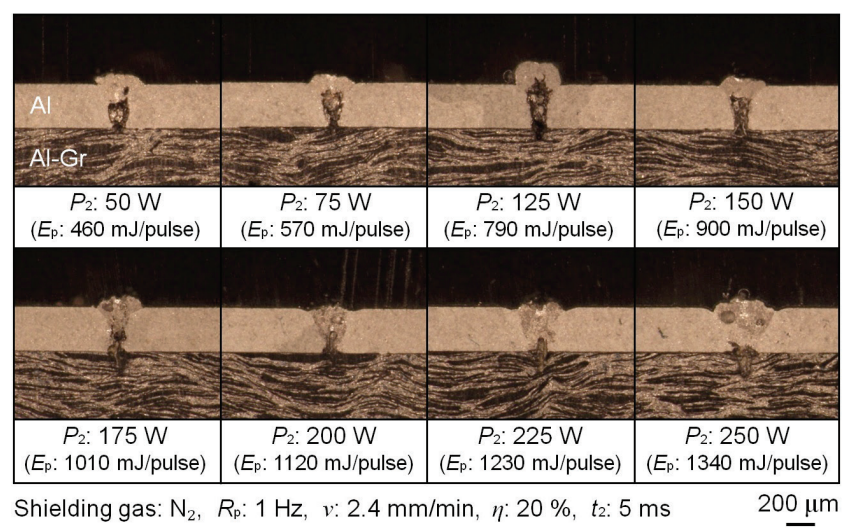

Fig. 7 Influence of laser power $\left(P_{2}\right)$ in phase 2 of spike pulse waveform.

priate value of laser power $P_{2}$ and time period $t_{2}$ at the phase 2 of spike pulse waveform.

In order to define an appropriate laser power for the phase 2 , the pulse width in phase $2\left(t_{2}\right)$ was kept constant at $5 \mathrm{~ms}$, while the laser power in phase $2\left(P_{2}\right)$ was varied between 50-250 W. From the cross-section observation as shows in Figure 7, it was seen that the porosity and bump have remained at a range of laser power 50-125 W, while an undercut was generated at the laser power more than $200 \mathrm{~W}$. The observation results of laser power $150 \mathrm{~W}$ and $175 \mathrm{~W}$ showed acceptable joining conditions, and $175 \mathrm{~W}$ was selected as the $P_{2}$ based on the deeper penetration and less porosity defect.

The next experiment was carried out to define an optimum pulse width to set the time period $t_{2}$ at the phase 2 of spike pulse waveform as shown in Figure 6. Based on the previous result, the $P_{2}$ was kept constant at $175 \mathrm{~W}$, while the $t_{2}$ was varied between $1.5-5.0 \mathrm{~ms}$. The pulse width was set less than $5 \mathrm{~ms}$ due to the limitation of maximum pulse width in the laser system used in this study. Figure 8 shows the cross-section views for various pulse widths under the same laser power of phase 2 to define the appropriate value of pulse width at the phase 2 for the spike pulse waveform. An appropriate irradiation time is necessary to melt and reduce a bump during a welding process. Within the range 1.5-2.5 ms of pulse width $\left(t_{2}\right)$, the results show that the welding defect of bump was not removed. Moreover, it could be cleared that the longer irradiation time ( $t_{2}: 3.5-5.0$ $\mathrm{ms}$ ) generated the larger size of bump. On the other hand,

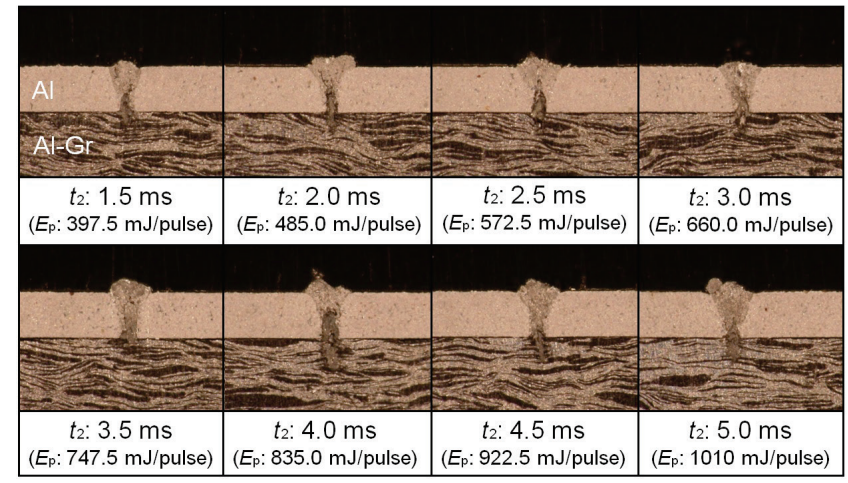

Shielding gas: $\mathrm{N}_{2}, R_{\mathrm{p}}: 1 \mathrm{~Hz}, v: 2.4 \mathrm{~mm} / \mathrm{min}, \eta: 20 \%, P_{2}: 175 \mathrm{~W} 200 \mu \mathrm{m}$

Fig. 8 Influence of pulse width $\left(t_{2}\right)$ in phase 2 of spike pulse waveform.

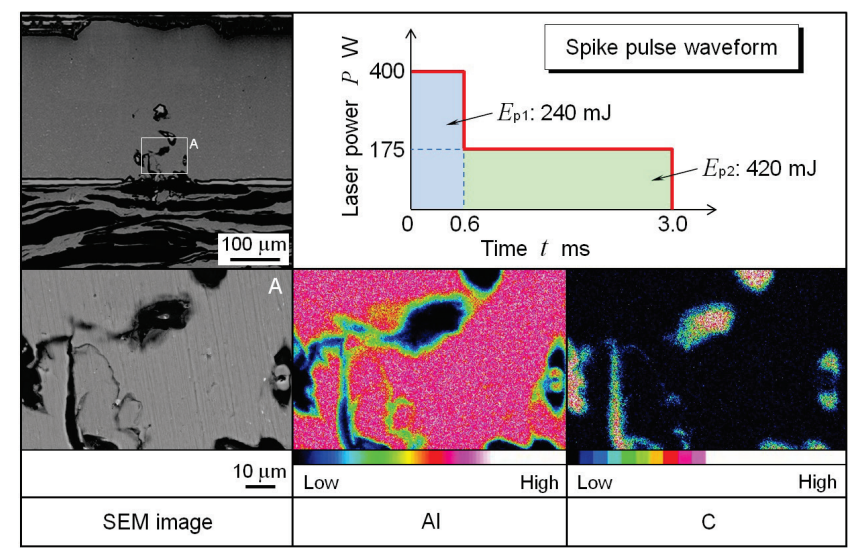

Fig. 9 SEM images and EDS mappings with spike pulse waveform.

the pulse width $t_{2}$ of 3 ms showed an acceptable penetration depth. Judging from these results, the $3 \mathrm{~ms}$ is selected as an appropriate value of $t_{2}$ at the phase 2 in the case of spike pulse waveform due to the stable penetration and smaller bump without porosity.

In addition, the elemental analysis was performed to observe the element distribution at the welded parts using a SEM equipped with the energy dispersive spectroscopy (EDS). Figure 9 showed the results of EDS mapping analysis in the case of spike pulse waveform. The shape of molten zone at the Al-Gr composite cannot be seen clearly because of too much carbon particles on the molten zone, which cannot be melted during the welding process. It could be seen that the coarse carbon located at the welded joint between pure aluminum and Al-Gr composite. It is considered that the severe convection of graphite particles with aluminum materials might be occurred in the molten zone. In other words, when Al-Gr composite is evaporated, an over-pressure is developed in the keyhole. This phenomenon would cause graphite particles pushed up from the prior zone in Al-Gr composite to the zone in the pure aluminum with melting phase, and finally the carbon particles would be redistributed during the re-solidification. However, it could be seen that a lack of fusion defect was generated by the spike pulse waveform. It could be detected by using an accurate microscopic observation in the high magnification condition. The lack of fusion defect in the molten zone can be identified by its string-like appearance, and it had randomly oriented curvature. 


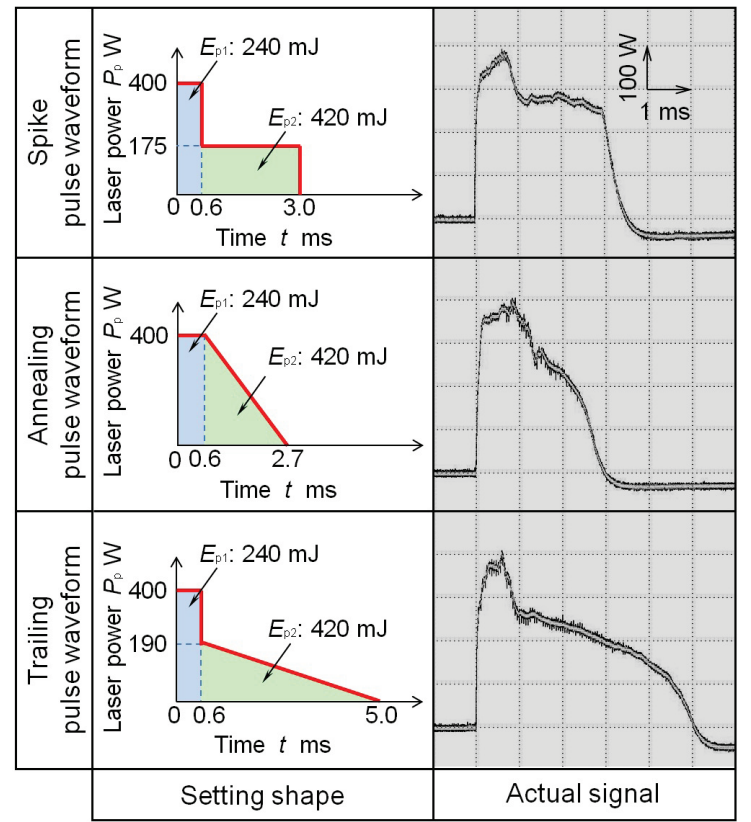

Fig. 10 Setting shape and actual signal of controlled pulse waveforms.
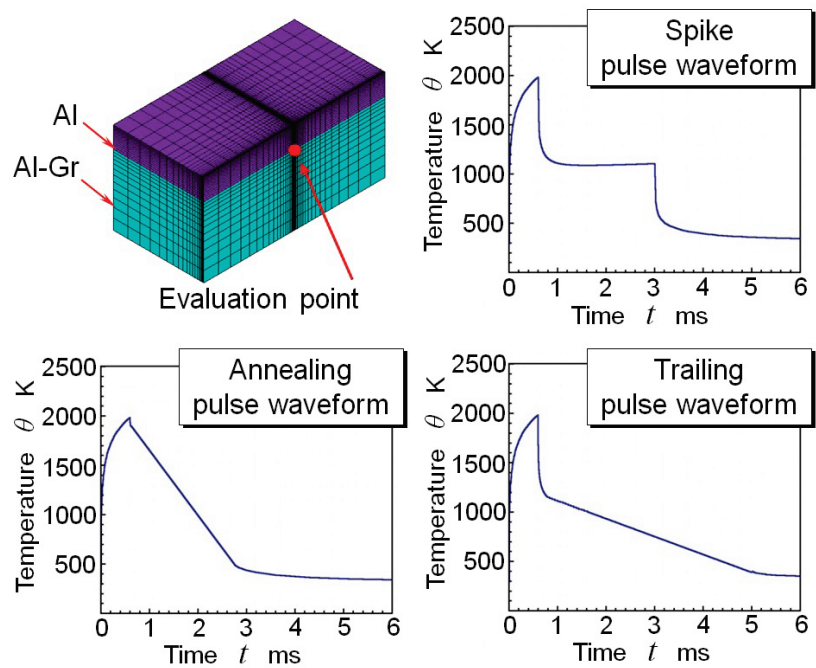

Fig. 11 Temperature histories by three controlled pulse waveforms.

From the observation results by the spike pulse waveform, the use of controlled pulse waveform has a positive effect on molten zone to remove the porosity and minimize the bump. However, the rapid cooling after welding process generated the coarse carbon particles and lack of fusion defect in the molten zone. Therefore, an improvement of heat input at the end of laser pulse is necessary by introducing an approach of ramp-down on the phase 2 to relieve the internal stress during re-solidification process and generate a better welded joint. The new controlled pulse waveforms are expressed as the annealing pulse waveform, and the trailing pulse waveform is also shown in Figure 10, in which the setting profile and actual signal of three controlled pulse waveforms are shown. Since the phase 2 of spike pulse waveform generates more stable weld bead compared with the rectangular pulse waveform, the amount

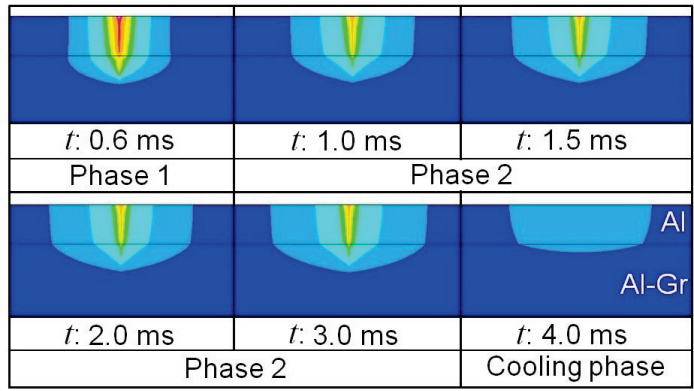

(a) Spike pulse waveform

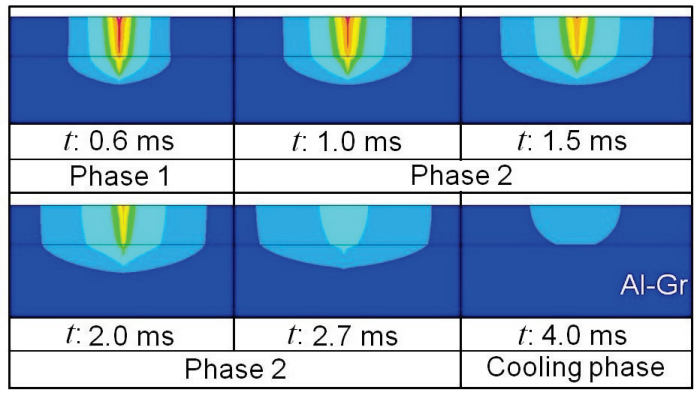

(b) Annealing pulse waveform

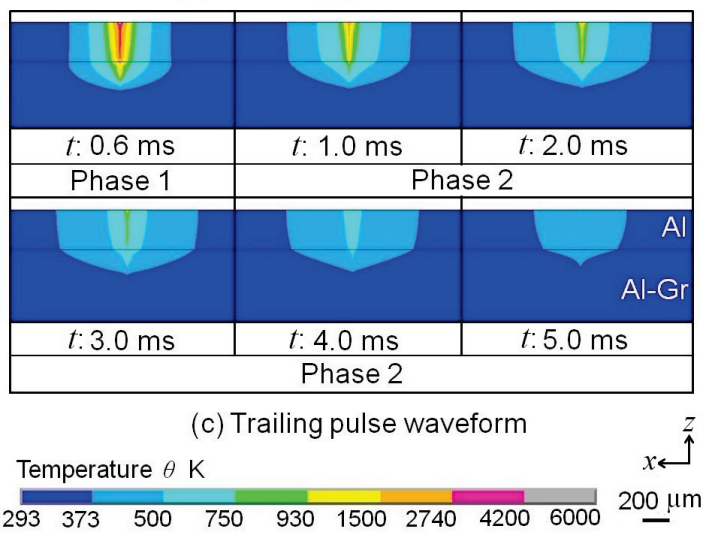

Fig. 12 Spatial temperature distribution by three controlled pulse waveforms.

of pulse energy at the phase 2 for the annealing and the trailing pulse waveforms was conducted under the same pulse energy of $420 \mathrm{~mJ}$. The main difference between the annealing and the trailing pulse waveforms are the laser power and irradiation time during the phase 2 . The annealing pulse waveform has the higher laser power with the shorter interaction time, while the trailing pulse waveform has the lower laser power with the longer interaction time.

The difference of temperature change by spike, annealing and trailing pulse waveforms were investigated with the thermal calculation. Spike, annealing and trailing pulse waveforms are finished at the time of $3.0 \mathrm{~ms}, 2.7 \mathrm{~ms}$ and $5.0 \mathrm{~ms}$, respectively. The temperature distributions were similar until $0.6 \mathrm{~ms}$ for three pulse waveforms, since they have similar pulse shape (laser power and pulse width) at the phase one. The main difference appeared at the pulse shape of phase 2 even under the same pulse energy $\left(E_{\mathrm{p} 2}\right.$ : $420 \mathrm{~mJ}$ ). Figure 11 and 12 show the temperature histories and distributions by these three controlled pulse waveforms. It can be seen that spike pulse waveform shows the constant temperature distribution during the phase 2 . Therefore, it is considered that the rapid cooling at $3 \mathrm{~ms}$ during the 


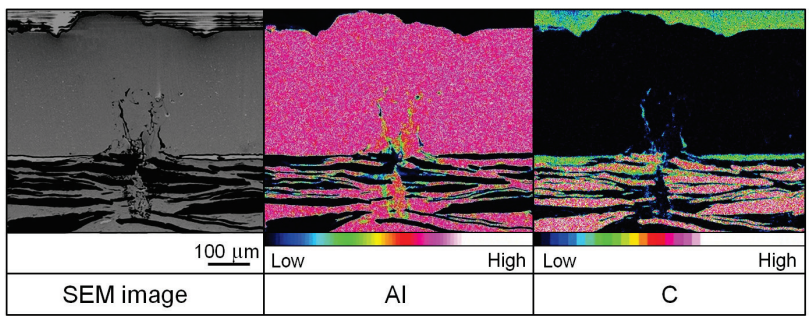

(a) Annealing pulse waveform

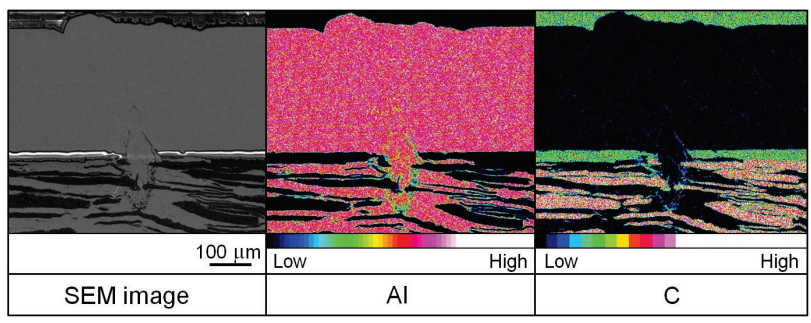

(b) Trailing pulse waveform

Fig. 13 SEM images and EDS mappings of cross-section with (a) annealing and (b) trailing pulse waveforms.

solidification generates internal stress, and it might cause the lack of fusion defect. From this disadvantage of spike pulse waveform, the slow cooling process is required during the solidification phase. As shown in the figures, the annealing pulse waveform shows the rapid decreasing of temperature distribution at the phase 2 . On the hand, the trailing pulse waveform indicates the gradual decreasing of temperature distribution at the phase 2 , which means that slow cooling could be realized. In other words, the longer time of laser irradiation is useful at the phase 2 in order to overcome the lack of fusion problem.

SEM images and EDS mappings of cross-section with annealing and trailing pulse waveforms are shown in Figure 13. In the SEM image, the shape of molten zone can be seen clearly if there are existences of aluminum element in the Al-Gr composite material. Compared with the spike pulse waveform (Figure 9), the size of carbon particles was much smaller in the case of annealing pulse waveform. However, it shows that the lack of fusion was appeared around the carbon particles. In the case of trailing pulse waveform, it can be seen that the specimens has been melted well with an acceptable weld bead state without lack of fusion defect. It also shows that a better weld bead state was obtained by applying the trailing pulse waveform. Furthermore, the sufficient long time during welding process, in other words, the slow cooling process during resolidification is necessary to avoid the appearance of carbon particles and minimize the lack of fusion defect in the molten zone.

\subsection{Evaluation of weld strength}

In order to evaluate the weld strength on the overlap welding with and without the control of pulse waveform under the constant laser pulse energy $\left(E_{\mathrm{p}}: 660 \mathrm{~mJ} / \mathrm{pulse}\right)$, the shearing test was carried out. The specimen for shear strength of overlap welding was designed with five seam lines and $1.5 \mathrm{~mm}$ distance between each line as shown in Figure 14. The shear strength was calculated by using fracture load and welding area. The value of shear strength was the average of three specimens. Then, the fracture surfaces were examined with SEM and EDS analysis.

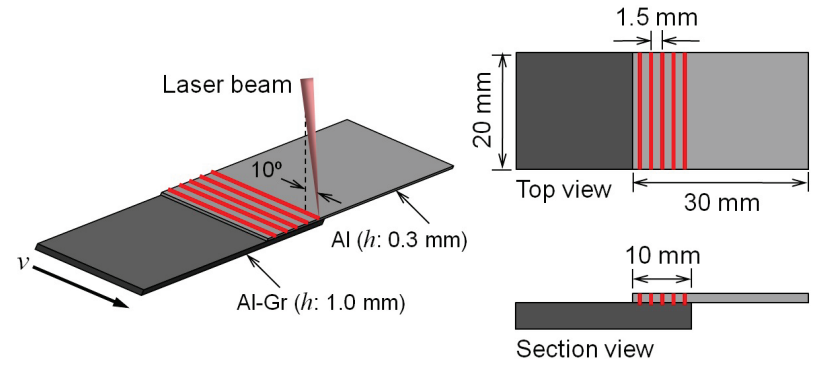

Fig. 14 Configuration of shearing test specimen.

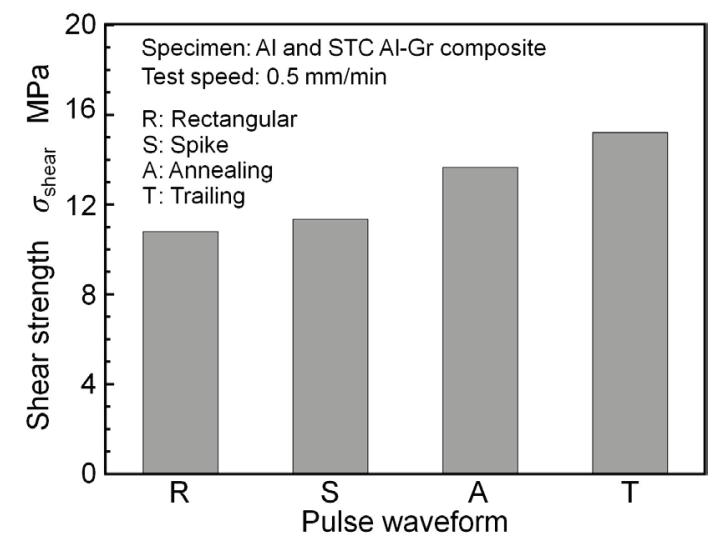

Fig. 15 Shear strength of the welding with and without the control of pulse waveforms.

Figure 15 shows the shear strength for various laser pulse waveforms. As shown in the figure, the weld joint with controlled trailing pulse waveform indicated the greater weld strength compared to the uncontrolled pulse waveform. The lower strength of the uncontrolled pulse waveform is attributable to the fact that the existence of porosity reduced the strength of weld joint. Meanwhile, it shows that the spike pulse waveform was less significant to increase the weld strength compared to the uncontrolled pulse waveform. It can be noted that the lack of fusion defect was affected on the lower weld strength. Therefore, it is cleared that the appropriate controlled laser pulse configurations are effective to improve the weld joint between aluminum and STC Al-Gr composite.

Figure 16 shows the fracture part on the top surface of Al-Gr composite after the shearing test. As can be seen from the figure, the fracture with uncontrolled pulse waveform occurred at the weld bead boundary in the Al-Gr composite and the presence of groove could be observed. It could be noticed that the existence of groove defect clearly influenced the weakness of weld joint strength. However, the fracture in the welding with the control of pulse waveform appeared inside the weld bead, which is located at the interface between pure aluminum and Al-Gr composite without the groove defect. In other words, the interfaces of aluminum and STC Al-Gr composite were expected to be the weak points of the weld joint. It is also considered that a weld joint without weld defects would increase the weld strength.

Figure 17 shows the side fracture surface of aluminum and STC Al-Gr composite, which were obtained from the shearing test under the welding condition with trailing pulse waveform. It can be seen that the fracture appearances show the brittle fracture. This fracture along the welding 


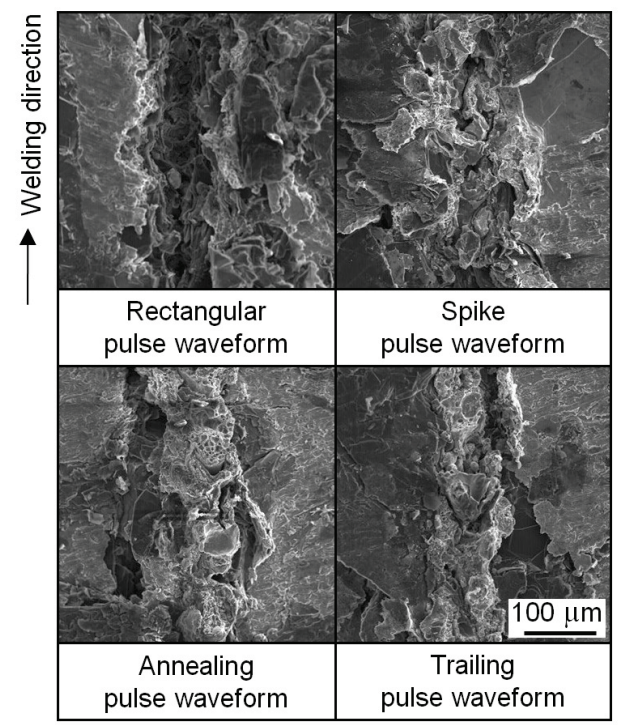

Fig. 16 SEM photographs of fracture on the top of Al-Gr composite.
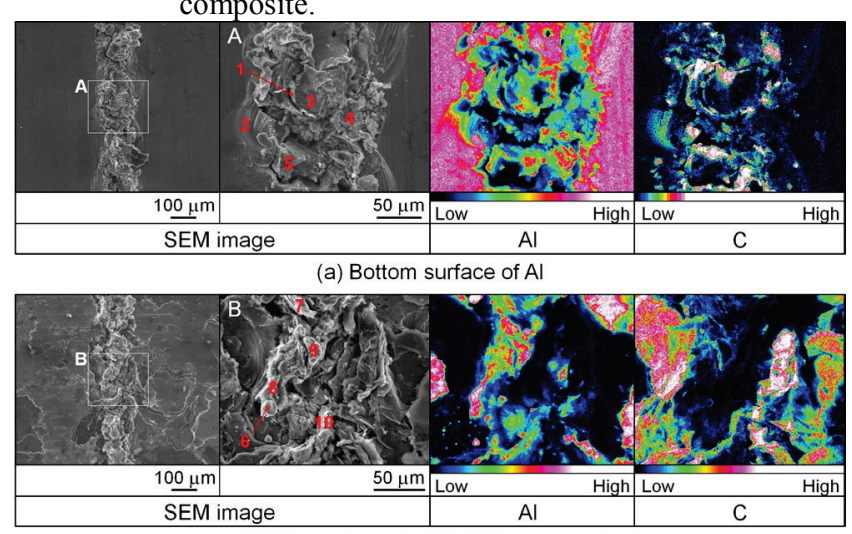

(b) Top surface of STC Al-Gr composite

Fig. 17 SEM photographs and EDS mappings of fracture with trailing pulse waveform.

Table 3 Element composition of points 1-10 in Figure 17

\begin{tabular}{c|c|c|c|c|c|c|c|c|c|c}
\hline Point no. & 1 & 2 & 3 & 4 & 5 & 6 & 7 & 8 & 9 & 10 \\
\hline $\mathrm{Al}$ (wt. \%) & 49.7 & 30.9 & 62.8 & 41.2 & 64.5 & 52.5 & 35.7 & 48.7 & 64.7 & 48.0 \\
\hline $\mathrm{C}$ (wt. \%) & 50.3 & 69.1 & 37.2 & 58.8 & 35.5 & 47.5 & 64.3 & 51.3 & 35.3 & 52.0 \\
\hline
\end{tabular}

interface would deteriorate the weld strength of aluminum and STC Al-Gr composite joint. Figure 17 also shows the distribution map of elements $\mathrm{Al}$ and $\mathrm{C}$ on the fracture zone, where spot analysis of points 1 to 10 are listed in Table 3. It can not be detected the aluminum carbide $\mathrm{Al}_{4} \mathrm{C}_{3}$ at the fracture zone, since the solid graphite can not be solidified into liquid aluminum. EDS mapping on the bottom surface of aluminum confirms that after the shearing test, the fine and coarse graphite particles were found sticks on melted aluminum without mixture with solidified aluminum.

In the case of fracture zone on the top surface of STC Al-Gr composite, it also can be seen that the solidified aluminum was squeezed out towards the edge of weld joint region, which showed the aluminum and graphite can not mixed together between both materials. Furthermore, the formation of the $\mathrm{Al}_{4} \mathrm{C}_{3}$ during welding process was successfully prevented during the welding process, which could deteriorate the strength of weld joint. In addition, the SEM observation revealed the crack was propagated in the fracture zone, which restricts the further strength of weld joint. However, compared with the bending strength of STC Al-Gr composite which is $7 \mathrm{MPa}$ in the thickness direction, the weld strength between the aluminum and STC Al-Gr composite showed the relatively higher strength. Therefore, it is clearly performed that the controlled laser pulse configurations are effective to produce a higher strength of joint for welding between an aluminum and STC Al-Gr composite.

\section{Conclusions}

The pulsed Nd:YAG laser micro-welding of a super thermal conductive (STC) aluminum-graphite composite by controlling the pulse waveform and heat input was experimentally and numerically investigated. Main conclusions obtained in this study are as follows:

(1) Porosity and bump were observed as remarkable weld defects in the overlap joining of $\mathrm{Al}$ and Al-Gr composite without a control of laser pulse.

(2) Proper control of laser power and pulse width could perform a positive result with largely free of weld defects and a relatively small bump.

(3) Controlled pulse waveform with slow cooling at the end of laser pulse was essential to relieve internal stress during solidification, since the lack of fusion was observed on the joining zone due to the rapid cooling.

(4) The higher shear strength could be obtained by the control of pulse waveform to perform a good joining without weld defects.

\section{Acknowledgments}

The authors are grateful to Shimane Institute for Industrial Technology for supplying super thermal conductive aluminum-graphite composites.

\section{References}

[1] N. Barekar, S. Tzamtzis, B.K. Dhindaw, J. Patel, N. Hari Babu and Z. Fan: Journal of Materials Engineering and Performance, 18, (2009) 1230.

[2] J. Niu, L. Pan, M. Wang, C. Fu and X. Meng: Vacuum, 80, (2006) 1396.

[3] P. Bassani, E. Capello, D. Colombo, B. Previtali and M. Vedani: Composites: Part A, 38, (2007) 1089.

[4] T. Ueno, T. Yoshioka, J. Ogawa, N. Ozoe, K. Sato and K. Yoshino: Synthetic Metals, 159, (2009) 2170.

[5] A.V. Kirillin and A.V. Kostanovskii: Physics-Uspekhi, 46, (2003) 1295.

[6] Brochure of Super Thermal Conductive Composites, Moriya Cutlery Laboratory, Ltd., (2010).

[7] E. Kannatey-Asibu: "Principles of Laser Materials Processing”, (John Wiley \& Sons, New Jersey, 2009) p. 233

(Received: June 06, 2011, Accepted: January 26, 2013) 